\title{
Optimal Dynamics of Functionality Development in Open Innovation
}

\author{
C. Watanabe ${ }^{*, * * *}$, J. Shin ${ }^{*}$, J. Heikkinen, ${ }^{* *}$ \\ A. Tarasyev \\ *Tokyo Institute of Technology, 2-12-1 Ookayama, Tokyo, Japan, \\ (e-mail:watanabe.c.aa@m.titech.ac.jp and shin.j.aa@m.titech.ac.jp) \\ ** University of Jyväskylä, P.O. Box 35 (Agora), Finland, \\ (e-mail: juheikki@jyu.fi) \\ *** IIASA (International Institute for Applied Systems Analysis), Laxenburg, Austria, \\ (e-mail:watanabe@iiasa.ac.atandtarasiev@iiasa.ac.at)
}

\begin{abstract}
Sustainable functionality development has become crucial option for firm's survival in a new paradigm confronting a post-information society. This can be realized by earlier emergence of functionality development. In this context, effective utilization of external innovation resources leads to follower substitution for leader in open innovation. This substitution induces advancements of innovative goods by substituting gratification of their consumption for resistance to them. Thus, optimization of utmost gratification of consumption under certain investment would be crucial for firm strategy. Optimal functionality development dynamics is analyzed in this paper by integrating production, diffusion and consumption functions, and using Pontryagin maximum principle. The result demonstrates that suprafunctionality can be developed through gratification of consumption substitution for resistance to new innovation.
\end{abstract}

Keywords: Optimal control, Optimal trajectory, Dynamics, Open innovation, Functionality development.

\section{INTRODUCTION}

In line with the advancement of informaton technology, the place where innovation takes place has been shifting from development site to diffusion site leading to a significant integration of production and diffusion functions as illustrated in Fig. 1 (Watanabe et al., 2005).

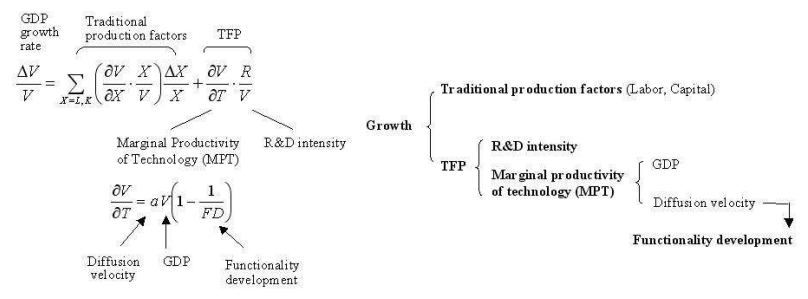

Fig.1. Options for growth.

Fig. 1 demonstrates that sustainable functionality development (FD) can be only the option for firm's competitiveness in a mature economy.

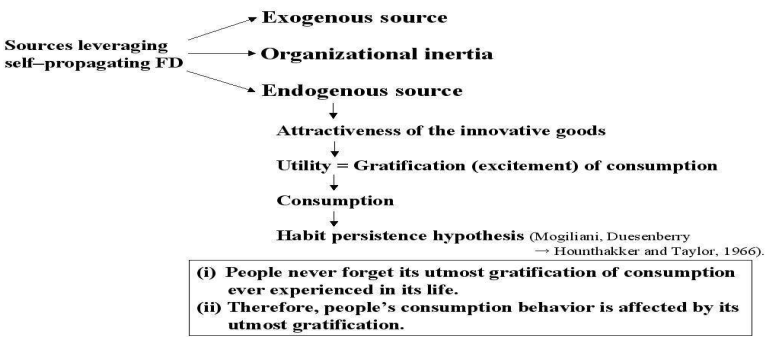

Fig. 2. Self-propagating FD leading to utmost gratification.
Fig. 2 illustrates possible options for sustainable FD which demonstrates that gratification of innovative goods consumption can lead to self-propagating FD.

Prompted by the foregoing hypothetical view, this paper demonstrates the significance of optimal FD dynamics for firm's sustainable FD.

Section 2 reviews FD in a diffusion trajectory. Section 3 analyzes requirements for sustainable FD. Optimal FD dynamics is demonstrated in Section 4. Section 5 briefly summarizes new findings and policy implications.

\section{FUNCTIONALITY DEVELOPMENT IN A DIFFUSION TRAJECTORY}

\subsection{The concept of functionality development}

Diffusion of innovative goods is induced by their ability to dramatically improve the performance of production processes, goods and services by means of innovation. This ability can be defined as functionality development (FD) (Watanabe et al., 2003). The emergence of innovation leads to new FD and the efforts for maintaining sustainable FD leads to creating successive innovations.

\subsection{Functionality development in a diffusion trajectory}

Diffusion of innovative goods terminates when FD obsolesces. Based on this postulate, Watanabe et al. (2004) demonstrated that the state of FD can be traced by the diffusion trajectory such as logistic growth model depicted as 
follows:

$$
\frac{d Y}{d t}=a Y\left(1-\frac{Y}{N}\right), \quad Y(t)=\frac{N}{1+b \exp (-a t)}
$$

where $Y$ : production of innovative goods; $N$ : carrying capacity; $a$ : velocity of diffusion; $b$ : initial state of diffusion; and $t$ : time trend.

Since degree of FD can be identified by potential capacity before reaching obsolescent stage, the degree of FD can be measured by the ratio of carrying capacity to the level of diffusion as follows:

$$
\text { Degree of } F D=N / Y=1+b \exp (-a t)
$$

Given the declining nature of FD as depicted by equation (2), firm's effort should focus on the prolongation of FD by means of effective utilization of potential resources in innovation. Such efforts can be identified by utilizing the Bass model (Bass, 1969) as it analyzes innovator $(p)$ and imitator $(q)$ dynamism in a diffusion process as depicted in equation (3).

$$
\frac{d Y}{d t}=(p N+q Y)\left(1-\frac{Y}{N}\right), \quad Y(t)=\frac{N(1-\exp (-(p+q) t))}{1+\frac{q}{p} \exp (-(p+q) t)}
$$

where $p$ : innovator parameter and $q$ : imitator parameter.

In the Bass model FD increases as the ratio of $q / p$ (the ratio of imitators $q$ and innovators $p$ ) increases since $d F D / d(q / p)>0$ (see Watanabe et al., 2008a), and thus $q / p$ can be identified as "prolongation ability".

Furthermore, Watanabe et al. (2008a) demonstrated that earlier FD emergence leads to sustainable FD as illustrated in Fig. 3. Therefore, next question is identification of the relationship between innovator and imitator by integrating the foregoing findings.

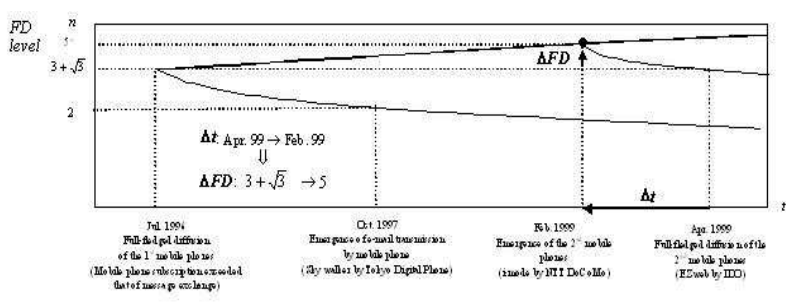

Fig. 3. Functionality development trajectory of the successive innovation in Japan's mobile phones (1996-2006).

\section{SUSTAINABLE FUNCTIONALITY DEVELOPMENT}

\subsection{Earlier emergence of functionality development}

Based on the analyses by Rogers (1983) and Mahajan et al. (1990), Moore (1991) and Watanabe et al. (2003) FD emergence can be identified as the ability to overcome the barrier of the market by means of new innovation. This level is equivalent to the level of $3+\sqrt{3}$ as illustrated in Fig. 4.

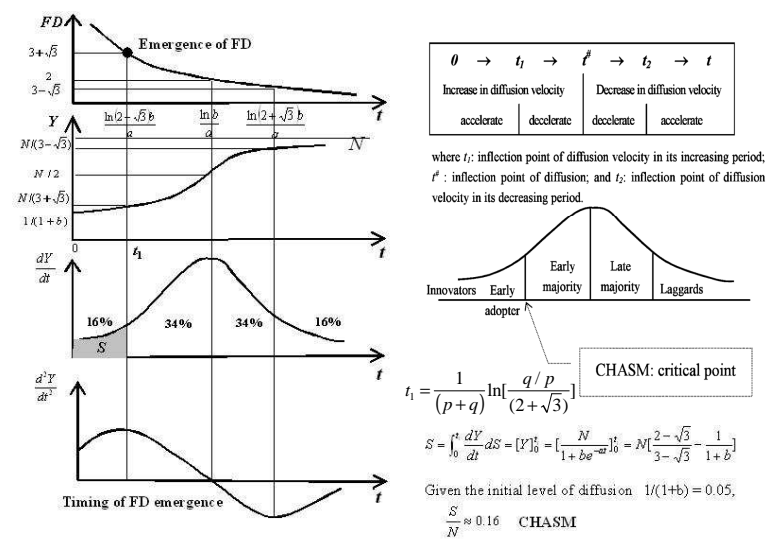

Fig. 4. Level and timing of inflection in a diffusion trajectory.

Moore (1991) pointed out that there exists a deep trench repelling new ventures start-up called CHASM. Consequently, it can be concluded that the emergence of FD occurs at certain point corresponding to cross the CHASM and also that the timing of FD emergence can be identified as the earlier inflection point of the first derivative of $t_{1}$.

\subsection{Governing factor accelerating functionality development emergence}

Firms' efforts in FD prolongation are managed by their technology strategy represented by their gross technology stock $T$. Generally, $T$ increases proportional to time $t$ as follows (see Watanabe et al., 2008b):

$T_{t}=\alpha+\beta t$

where $\alpha$ and $\beta(\alpha, \beta>0)$ : coefficients.

Therefore, $Y(t)$ can be depicted as

$Y(t)=\frac{N}{1+b \exp (-a t)}=\frac{N}{1+b^{\prime} \exp \left(-a^{\prime} T_{t}\right)}$,

where $a^{\prime}$ and $b^{\prime}$ : coefficients for velocity of diffusion driven by $T_{t}$, and initial state of diffusion, respectively.

Thus, diffusion trajectory indicated by $t$ can by depicted as a function of $T$. With the foregoing understanding, FD function can be developed to multi-logistic growth model as a function of gross technology stock $T$ as depicted by the following equation:

$$
\begin{aligned}
F D(t) & =\frac{N}{Y(t)}=1+b^{\prime} \exp \left(-a^{\prime} T_{t}\right) \approx 1+b^{\prime}\left(1-a^{\prime} T_{t}\right) \\
& =\sum_{j=1}^{n}\left(1+b_{j}\left(1-a_{j} T_{t}\right)\right)=\sum_{j=!}^{n} F D_{j}\left(T_{t}\right),
\end{aligned}
$$

where $a_{j}=\frac{a^{\prime} b^{\prime}}{b_{j}}, b_{j}=\left(b^{\prime}-(n-1)\right) P_{j}, \sum_{j=1}^{n} P_{j}=1$.

These postulates can be applied also to the Bass model as it can be decomposed to the logistic growth model. Gross technology stock $T$ consists of indigenous technology stock 
$T_{i}$ and assimilated spillover technology as $T=T_{i}+z T_{s}$, where $z$ : assimilation capacity; $T_{i}$ : indigenous technology ; and $T_{s}$ : technology spillover pool. Level of gross technology stock $(T)$ increases in a cascading way depending on assimilation capacity ( $z)$ as illustrated in Fig. 5.

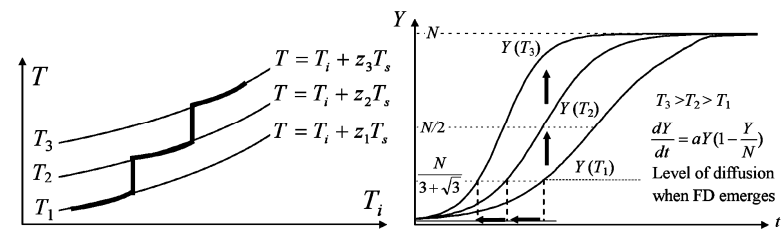

Fig. 5. Acceleration of FD emergence depending on assimilation capacity and diffusions trajectory.

Therefore, its level differs depending on the level of $z T_{s}$, particularly the level of $z$ given the exogenously governed nature of $T_{s}$. Fig. 3. indicates that higher level of diffusion also enables earlier timing of the emergence of FD with higher level. As reviewed earlier, since level of $T$ increases in a cascading way depending primarily on assimilation capacity $z$, and timing of FD emergence is accelerated as $T$ increases, level of FD can be classified into three categories depending on assimilation capacity $z$ conditions as (i) Decreasing FD $(z=0)$, (ii) Constant FD $\left(d z / d T_{i}=0\right)$, and (iii) Sustainable FD $\left(d z / d T_{i}>0\right)$ as illustrated in Fig. 6.

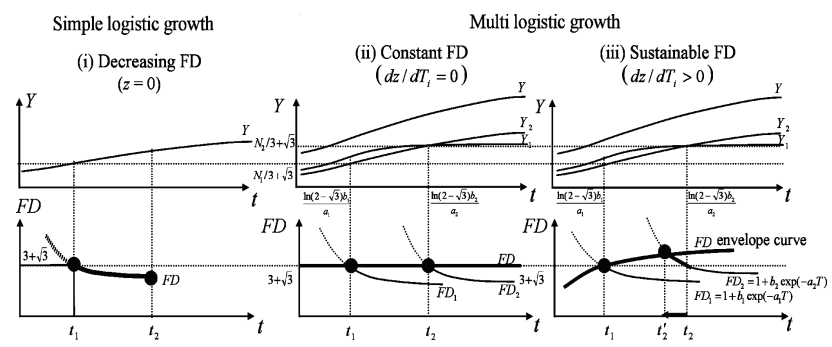

Fig. 6. Comparison of growth trajectory between simple and multi-logistic growth.

Self-propagating assimilation capacity increase leads to sustainable FD. The third case "Sustainable FD" can be attributed to self-propagating assimilation capacity increase leading to prolongation ability of FD. This corresponds to prolongation ability $q / p$ increase in the Bass model. Since FD enhances corresponding to the higher level of $T$, in case of a co-evolutionary dynamism between $z T_{s}$ and $T$ ( $T$ enhances as $z T_{s}$ increases which in turn induces further $z T_{s}$ ), multi-logistic growth leads to sustainable FD trajectory. Level of diffusion increases as assimilated spillover technology increases leading to higher level of FD at its emergence. This trajectory can be traced as an envelope curve of FD. Sustainable FD can be anticipated when the level of FD that emerged in the successive wave enhances than the level of preceding wave. This can be expected when assimilated spillover technology increases as innovation advances.

\subsection{Requirement for earlier functionality development emergence}

Aiming at demonstrating sustainable FD by means of earlier emergence of FD, an empirical analysis taking the diffusion trajectory of high-technology in the Bass model is conducted. Since $q / p \equiv x$ demonstrates prolongation ability in FD, the following analysis examines whether increasing $x$ contributes to decrease in $t_{l}$ (the timing of FD emergence). Differentiating $t_{l}$ in Fig. 4 with respect to $q / p \equiv x$,

$\frac{d t_{1}}{d q / p}=\frac{d t_{1}}{d x}=\frac{d v}{d x} \ln (x /(2+\sqrt{3}))+\frac{v}{x}$,

where $v=\frac{1}{p(1+x)}, \frac{v}{x}=\frac{1}{p(1+x) x}$ and $\frac{d v}{d x}=\frac{-\left[(1+x) \frac{d p}{d x}+p\right]}{[p(1+x)]^{2}}$.

Thus, the condition to accelerate FD emergence by $q / p$ increase is:

$$
\begin{aligned}
& \frac{d t_{1}}{d x}=\frac{1}{p x(1+x)}\left[1+\frac{\left[(1+x) \frac{d p}{d x}+p\right] \ln \left[\frac{(2+\sqrt{3})}{x}\right] x}{p(1+x)}\right]<0 \\
& \Leftrightarrow W(x) \equiv \frac{\left[(1+x) \frac{d p}{d x}+p\right] \ln \left[\frac{(2+\sqrt{3})}{x}\right] x}{p(1+x)}<-1 .
\end{aligned}
$$

From the above analysis, it can be concluded that inequality (9) depicts the necessary condition for earlier FD emergence.

Based on the previous subsection, the boundary condition satisfying earlier FD emergence in the Bass model can be developed as follows:

$\frac{d t_{1}}{d x}<0 \Leftrightarrow W(x)<-1 \Leftrightarrow \frac{\frac{d p}{d x} \ln \left[\frac{(2+\sqrt{3})}{x}\right] x}{p}+\frac{\ln \left[\frac{(2+\sqrt{3})}{x}\right] x}{(1+x)}<-1$.

Analyzing cases $1<x<2+\sqrt{3}$ and $x>2+\sqrt{3}$ separately leads to following two inequalities:

$\frac{d t_{1}}{d x}<0 \Leftrightarrow \begin{cases}\varepsilon<B(x), & 1<x<2+\sqrt{3} \\ \varepsilon>B(x), & x>2+\sqrt{3},\end{cases}$

where $\varepsilon=\frac{d p}{d x} \frac{x}{p}, \quad B(x)=-\frac{1}{\ln ((2+\sqrt{3}) / x)}-\frac{x}{(1+x)}$ is a boundary

function. The areas satisfying conditions for $d t_{1} / d x<0$ are illustrated in Fig. 7.

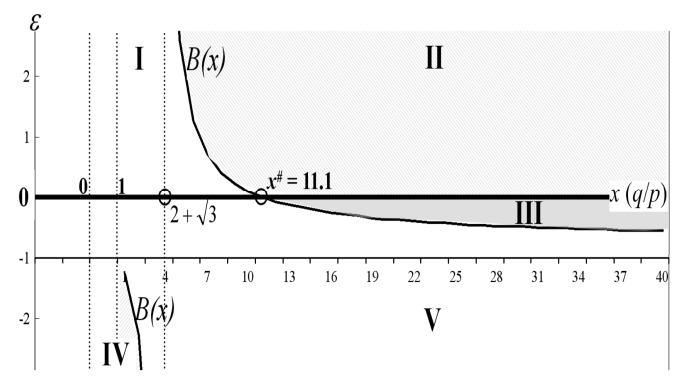

Fig. 7. Boundary function.

Furthermore, in order to accomplish open innovation, which means shifting from $p$ to $q$, the condition $d p / d x<0$ should also be satisfied. The area which satisfies both conditions $d p / d x<0$ 
and $d t_{1} / d x<0$ with the value of $q / p$ larger than 11.1 (the crossing point of boundary function $B(x)$ is $x^{\#}=11.1$ ) can be identified.

\subsection{Requirement for sustainable functionality development (Imitator substitutes for innovator)}

Given the $x$ is large enough, $B(x) \approx-1$ and the necessary condition for $d p / d x<0$ and $d t_{1} / d x<0$ (area III in Fig. 7.) is

$-1<\frac{d p}{d x} \frac{x}{p}<0 \Leftrightarrow \frac{d \ln x}{d \ln p}<-1 \Leftrightarrow \frac{d \ln q}{d \ln p}<0 \Leftrightarrow \frac{d q}{d p}<0$.

This inequality suggests the substituting relationship between $p$ and $q$. Furthermore, the substituting dynamism between $p$ and $q$ can be identified as follows:

$$
\begin{aligned}
& \frac{d p}{d q / p}<0 \Leftrightarrow \frac{d q / p}{d p}<0 \Leftrightarrow \frac{d q / p}{d p} \cdot \frac{p}{q / p}<0 \\
& \Leftrightarrow \frac{d \ln q}{d \ln p}<1 \Leftrightarrow \frac{\Delta q / q}{\Delta p / p}<1 .
\end{aligned}
$$

Since $\Delta p / p<0, \frac{\Delta q / q}{\Delta p / p}\left(-\frac{\Delta p}{p}\right)<\left(-\frac{\Delta p}{p}\right)$

$$
\Leftrightarrow-\frac{\Delta q}{q}<-\frac{\Delta p}{p} \Leftrightarrow \frac{\Delta q}{q}>\frac{\Delta p}{p} \Rightarrow p \rightarrow q .
$$

Therefore, substitution direction can be identifies as from $p$ to $q$. Stimulated by the foregoing observations, sustainable FD through earlier emergence of FD depends on follower substitution for leader. This substitution corresponds to the dynamism triggered by open innovation. Consequently, it can be concluded that follower substitution for leader leverages the sustainable FD in open innovation.

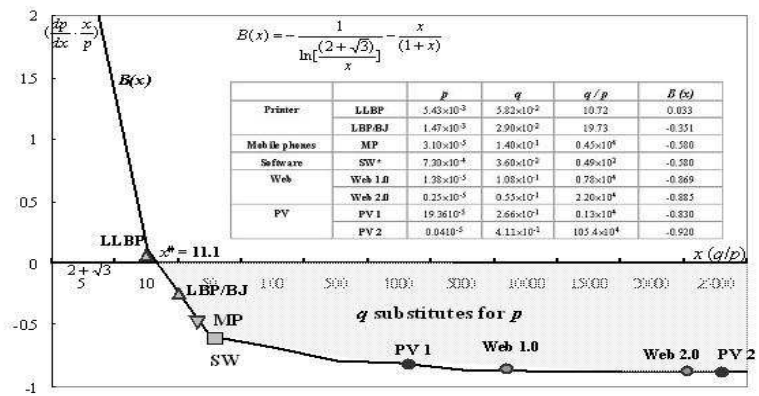

Fig. 8. Sustainable FD condition.

This substitution induces consumption of innovative goods by gratification substitution for resistance to new technology.

\section{OPTIMAL CONTROL PROBLEM}

On the basis of the findings obtained in the preceding section, optimization of utmost gratitude of innovative goods consumption under certain investment constraints is analyzed. This section proposes a corresponding optimal control problem with the infinite horizon and solves it using the Pontryagin maximum principle (Pontryagin et al., 1962). In this problem, the control variable is investment intensity in the production factors, and the utility function is the integral discounted consumption index. Applying the Pontryagin maximum principle, the optimal investment policy and the corresponding trajectories of the FD, which maximize the utility function, can be observed. An empirical analysis focusing on the diffusion trajectory of Japan's mobile phone is conducted. On the basis of empirical analyses, assessment of the optimal level of FD is conducted.

\subsection{Model's main variables}

In order to analyse the optimal level of FD, the following main variables can be demonstrated:

$t \in\left[t_{0},+\infty\right)-$ Time on the infinite horizon;

$Y=Y(t)-$ Production;

$N=N(t)-$ Carrying capacity;

$F D=F D(t)=\frac{N(t)}{Y(t)}-$ Functionality development;

$\eta=\eta(t)=\frac{Y(t)}{N(t)}=\frac{1}{F D(t)}-$ Production to carrying capacity;

$s=s(t)=\frac{\dot{N}(t)}{Y(t)}-$ Investment intensity.

4.2 System's dynamics

$\dot{Y}=a \cdot Y(t) \cdot(1-\eta(t))$

$\dot{\eta}=a \cdot \eta(t) \cdot(1-\eta(t))-\eta^{2}(t) \cdot s(t)$

This dynamics of innovation productivity can be expressed as follows:

$\dot{\eta}=a \cdot \eta(t) \cdot\left(1-\eta(t)-\frac{s(t)}{a} \cdot \eta(t)\right)$

Let $s(t)=s_{0}>0$,

$\dot{\eta}=a \cdot \eta(t) \cdot\left(1-\eta(t)-\frac{s_{0}}{a} \cdot \eta(t)\right) \cdot$

Based on this, stationary point can be depicted as follows:

$\begin{array}{ll}1-\eta-\frac{s_{0}}{a} \cdot \eta=0 & 0<\eta=\left(\frac{a}{a+s_{0}}\right)<1 \\ 1-\eta \cdot\left(\frac{a+s_{0}}{a}\right)=0 & \end{array}$

\subsection{Optimal control problem}

$\theta(t)=F D(t)-1 \Leftrightarrow F D(t)=\theta(t)+1 \Leftrightarrow \dot{F D}(t)=\dot{\theta}(t)$

where $\theta$ : the first phase variable; $Y$ : the second phase variable; and $s$ : investment control.

$\dot{\theta}(t)=s(t)-a \cdot \theta(t), \theta\left(t_{0}\right)=\theta^{0}, 0 \leq s(t) \leq A<1$ 
$\dot{Y}(t)=a \cdot Y(t) \cdot\left(\frac{\theta(t)}{\theta(t)+1}\right), Y\left(t_{0}\right)=Y^{0}$

\subsection{Utility function (Integrated Logarithmic Consumption} Index)

Consumption $=Y(t) \cdot(1-s(t))$.

$J(\theta(t), y(t), s(t))=\int_{0}^{+\infty} e^{-\rho \cdot t} \cdot(\ln Y(t)+\ln (1-s(t))) d t$

where $e^{-\rho \cdot t}$ : discount factor.

The optimality is understood with respect to the utility function $J$ represented by an integral with a discount coefficient $\rho$.

Adjoint variables

$\psi_{1}=\psi_{1}(t)=e^{\rho t} \psi_{1}^{*}(t)$ and $\psi_{2}=\psi_{2}(t)=e^{\rho t} \psi_{2}^{*}(t)$ - Prices of production and functionality development.

\subsection{Stationary Hamiltonian}

Application of the Pontryagin maximum principle

Hamiltonian problem which measures the current flow of utility from all sources is depicted in equation (23).

$H\left(\theta, Y, \psi_{1}, \psi_{2}, s\right)=\ln Y+\ln (1-s)+\psi_{1} \cdot(s-a \cdot \theta)+\psi_{2} \cdot a \cdot Y \cdot \frac{\theta}{\theta+1}$

where $\psi_{1}$ : shadow price for $\theta$, and $\psi_{2}$ : shadow price for $Y$. ( $\psi_{1}$ and $\psi_{2}$ : adjoint variables).

Necessary condition of maximum for Hamiltonian function

$\frac{\partial H}{\partial s}=-\frac{1}{1-s}+\psi_{1}=0 \Leftrightarrow s=1-\frac{1}{\psi_{1}}=\frac{\psi_{1}-1}{\psi_{1}}$

Maximized Hamiltonian

$H\left(\theta, Y, \psi_{1}, \psi_{2}\right)=\ln Y-\ln \psi_{1}+\psi_{1} \cdot\left(1-\frac{1}{\psi_{1}}-a \cdot \theta\right)+\psi_{2} \cdot a \cdot Y \cdot \frac{\theta}{\theta+1}$

Adjoint equations (Dynamics of prices)

$\dot{\psi_{1}}=\rho \cdot \psi_{1}(t)-\frac{\partial H\left(\theta(t), Y(t), s(t), \psi_{1}(t), \psi_{2}(t)\right)}{\partial \theta}=\rho \cdot \psi_{1}(t)+a \cdot \psi_{1}(t)-a \cdot \frac{\psi_{2}(t) \cdot Y(t)}{(\theta(t)+1)^{2}}$

$\dot{\psi}_{2}=\rho \cdot \psi_{2}(t)-\frac{\partial H\left(\theta(t), Y(t), s(t), \psi_{1}(t), \psi_{2}(t)\right)}{\partial Y}=\rho \cdot \psi_{2}(t)-a \cdot \frac{\psi_{2}(t) \cdot \theta(t)}{(\theta(t)+1)}-\frac{1}{Y(t)}$

where $\dot{\psi}_{1}$ : changing price for $\theta$, and $\dot{\psi}_{2}$ : changing price for $Y$.
Cost variables

$C_{1}=C_{1}(t)=\psi_{1}(t) \cdot \theta(t)$, and $C_{2}=C_{2}(t)=\psi_{2}(t) \cdot Y(t)$.

Dynamics of cost variables

$\dot{C}(t)=\dot{\psi}_{1}(t) \cdot \theta(t)+\psi_{1}(t) \cdot \dot{\theta}(t)+\dot{\psi}_{2}(t) \cdot Y(t)+\psi_{2}(t) \cdot \dot{Y}(t)$

Stationary equations of the Hamiltonian system

Stationary points of the Hamiltonian system

$1-\frac{\theta}{C_{1}}-a \cdot \theta=0, \rho \cdot C_{1}-\frac{1}{\rho} \cdot \frac{a \cdot \theta}{(\theta+1)^{2}}+\frac{C_{1}}{\theta}-1=0 \quad C_{2}=\frac{1}{\rho}$

Solution of stationary equations of the Hamiltonian system: the first equation

$C_{1}=\frac{\theta}{(1-a \cdot \theta)}$, and $\theta=\frac{C_{1}}{a \cdot C_{1}+1}$.

Solution of stationary equations of the Hamiltonian system: the second equation

$\frac{(a+\rho) \cdot \rho}{a} \cdot C_{1}=\frac{\theta}{(\theta+1)^{2}}$

Solution of stationary equations for FD

$\frac{(a+\rho) \cdot \rho}{a}=\frac{1-a \cdot \theta}{(\theta+1)^{2}}$

Consequently, based on these analyses, optimal level of FD can be depicted as follows :

$F D=\frac{a}{2 \cdot(a+\rho) \cdot \rho} \cdot\left(-a+\left(a^{2}+4 \cdot \frac{(a+\rho) \cdot \rho \cdot(a+1)}{a}\right)^{\frac{1}{2}}\right)$

4.6. Optimal dynamics of FD leading to supra-functionality emergence

On the basis of equation (29), trajectory under certain investment intensity that maximizes utility function leading to utmost gratification of consumption was analyzed. Fig. 9 compares optimal and actual level of FD in Japan's mobile phone development trajectory over the last decade.

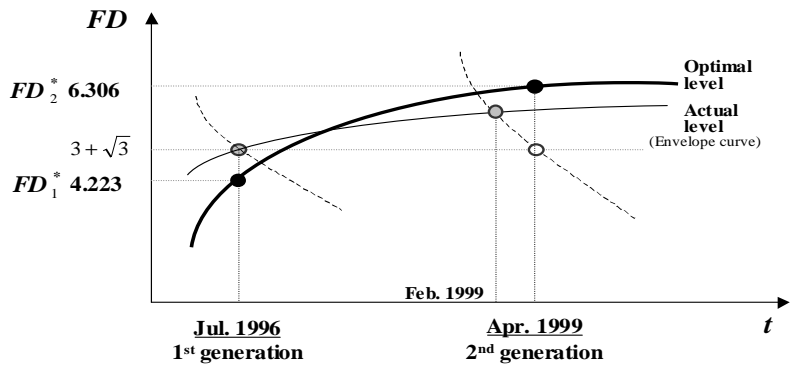

Fig. 9. Comparison of optimal and actual levels of FD in Japan's mobile phones development trajectory (1996-2006). 
This optimal level corresponds to "supra-functionality," which includes social, cultural, aspirational, and emotional needs. If the innovative goods do not resonate with the above demand, a psychological barrier may develop impeding the customers "affinity" with the innovation resulting in its abandonment (D. McDonagh, 2008).

Japan's mobile phone development trajectory over the last decade demonstrates this dynamics suggesting a possibility of follower (optimal level) substitutes for leader (actual level) in open innovation as demonstrated in Fig. 10.

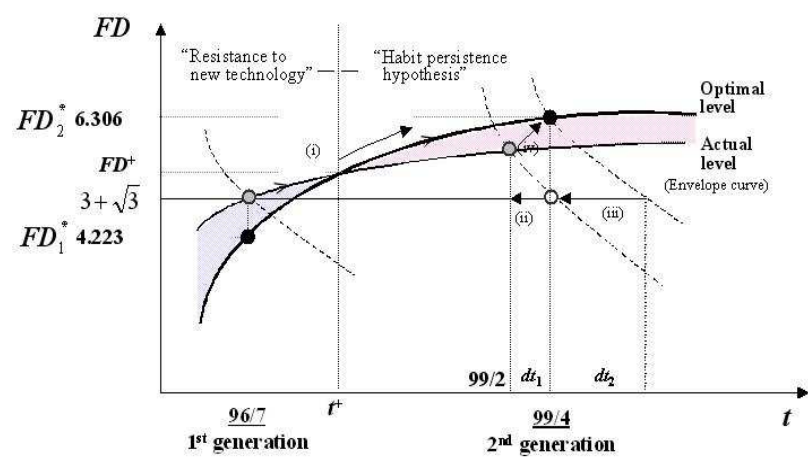

Fig. 10. Dynamism leading to supra-functionality in Japan's mobile phones development (1996-2006).

Looking at Fig. 10, we note the following implications:

(i) Shift from "Resistance to new technology" to "Habit persistence hypothesis" at utmost FD level $\left(\mathrm{FD}^{+}\right)$.

(ii) Follower substitutes leader $\frac{d t_{1}}{d q / p}<0$

(iii) Acceleration of follower substitutes leader $\frac{d t_{2}}{d q / p}<0$

(iv) Leverage by "Habit persistence hypothesis" anticipating supra-functionalty.

\section{CONCLUSIONS}

In light of the significance of the sustainable FD for firms' profitability in their innovation, this paper analyzed necessary conditions enabling firms prolong their FD and the optimal level of FD leading to supra-functionality.

Noteworthy findings obtained include:

(i) Sustainable FD for sustainable growth under the constraint economy.

(ii) Sustainable FD can be anticipaed by the early emergence of FD.

(iii) Early emergence of FD can be enabled by $q$ (follower) substitution for $p$ (leader) corresponding to open innovation.

(iv) Optimal dynamics of FD corresponds to the maximization of gratification of consumption under certain investment intensity leading to suprafunctionality.

(v) This dynamism corresponds to the 'supra-functionality' (follower) substitution for 'Resistence to new technology' (leader).

\section{REFERENCES}

Bass, F.M. (1969). A new product growth model for consumer durables. Management Science, 15 (5), 215227.

Bauer, M. (1995). Resistance to new technology. Cambridge university press.

Kryazhimskiy, A.V., Watanabe, C. (2004). Optimization of technological growth. GENDAITOSHO, Kanagawa.

Mahajan, V., Muller, E., and Srivastara, R.K. (1990). Determination of adopter categories by using innovation diffusion models. Journal of Marketing Research, 27, 37-50.

McDonagh, D. (2008). Satisfying needs beyond the functional: The changing needs of the silver market consumer. Proceedings of the International Symposium on the Silver Market Phenomenon - Business Opportunities and Responsibilities in the Aging Society, Tokyo.

Meyer, P.S., and Ausbel, J.H. (1999). Carrying capacity: A model with logistically varying limits. Technological Forecasting and Social Change, 61 (3), 209-214.

Moore, G.A. (1991). Crossing the Chasm. New York, Harper Business Essentials.

Pontryagin, L.S., Boltyanskii, V.G., Gamkrelidze, R.V., Mishchenko, E.F. (1962). The Mathematical Theory of Optimal Processes, Interscience, New York.

Rogers, E.M. (1983). Diffusion of innovation. 3rd ed. The Free Press, New York.

Tarasyev, A.M., and Watanabe, C. (2001). Dynamic optimality principles and sensitivity analysis in models of economic growth, Nonlinear Analysis, 47 (4), 23092320 .

Watanabe, C., Asgari, B., and Nagamatsu, A. (2003). Virtuous cycle between R\&D, functionality development and assimilation capacity for competitive strategy in Japan's high-technology industry. Technovation, 23 (11), 879-900.

Watanabe, C., Kondo, R., Ouchi, N., Wei, H., and GriffyBrown, C. (2004). Institutional elasticity as a significant driver of IT functionality development. Technological Forecasting and Social Change, 71 (7), 723-750.

Watanabe, C., Hur, J.Y., and Matsmoto, K. (2005). Technological diversification and firm's technoeconomic structure: An assessment of Canon's sustainable growth trajectory. Technological Forecasting and Social Change, 72 (1), 11-27.

Watanabe, C., Moriyama, K., and Shin, J. (2008a). Functionality development dynamism in a diffusion trajectory: A case of Japan's mobile phone development. Technological Forecasting and Social Change, in print.

Watanabe, C., Shin, J., and Heikkinen, J. (2008b). Follower substitution for a leader as a source of new functionality development in open innovation. IEEE TMC-Japan, 4853 . 